\title{
CONSTRUÇÃO DO GUIA DE PADRÕES DE CRIATIVIDADE PARA DESENVOLVEDORES DE SOFTWARE
}

\author{
Elton R. Vieira \\ Universidade Federal de Pernambuco \\ (eltonrvs@gmail.com)
}

\begin{abstract}
Resumo: Técnicas de Criatividade são ferramentas utilizadas para o estímulo do pensamento criativo, auxiliando pessoas a encontrar soluções inovadoras para seus problemas. A importância da criatividade no desenvolvimento de software é reconhecida e estudada por pesquisadores nas últimas décadas, contudo desenvolvedores de software ainda fazem pouco uso dessas ferramentas, a detrimento de suas vantagens. Com o objetivo de encorajar a adoção e o suporte ao uso das técnicas de criatividade no desenvolvimento de software, foi revisado e reestruturado um grande número de técnicas e criado o Guia de Padrões de Criatividade. O Guia segue os princípios de Padrões de Projeto, explicando e exemplificando o uso das técnicas de criatividade no contexto de desenvolvimento de software. Este artigo descreve o desenvolvimento do Guia, as escolhas metodológicas, estrutura das páginas e a interface final da ferramenta.
\end{abstract}

Palavras-chave: Técnicas de Criatividade, Engenharia de Software, Inovação, Padrões de Projeto.

\begin{abstract}
Creativity techniques are tools for stimulating creative thinking, which can help people finding innovative solutions for their problems. The importance of creativity fostering techniques in software development has been recognized and investigated by researchers for over a decade, yet the greater software engineering community makes little use of the myriads of techniques available. In order to encourage a wider adoption and to support the use of creativity techniques in software development, we have reviewed a large number of creativity techniques and have created a Creativity Patterns Guide. The guide follows the familiar concepts of Design Patterns, explaining and exemplifying the use of creativity techniques in the context of software development. This paper describes the development of the Creativity Patterns Guide, the mmetodologial choises, pages structures and final interface.
\end{abstract}


Keywords: Creativity Techniques, Software Engineering, Innovation, Design

Patterns.

\section{INTRODUÇÃO}

O processo de desenvolvimento de um software é muitas vezes complexo e repletos de problemas para serem solucionados, desde a fase da ideia original, até a fase de teste e implantação. Muito desses problemas, incluindo problemas de design e arquitetura da informação, podem ser resolvidos criativamente, resultando em inventivos softwares, design de sistemas criativos, ou até produzindo um inovador e efetivo algoritmo de programação.

Sabendo da necessidade atual de conceitos inovadores no mercado de software, o exercício da criatividade é particularmente importante nessa área. Identificar as necessidades do usuário, desenvolver novas ferramentas e inovadores serviços é passo importante para permanecer atendendo e construindo demandas para os usuários (POTTS, 1995; ALVES, RAMALHO e DAMASCENO, 2007). O desenvolvimento de software pode envolver múltiplos stakeholders, equipe multidisciplinar e situações dinâmicas. Conduzir esta complexa atividade também requer criatividade (SÓLIS e ALI, 2010).

Pesquisas sugerem que tais problemas podem ser combatidos utilizando técnicas de estímulo à criatividade (FOSTER, 2008; MUNARI, 1998; NORMAN, 2004). Há um grande número de técnicas de criatividade na literatura (BONFIM, NAGEL e ROSSI, 1997; MYCOTED, 2014; MIND TOOLS, 2014), alguns já estudados no contexto de desenvolvimento de software, apresentando bons resultados (SÓLIS e ALI, 2010; NGUYEN e SHANKS, 2008; MAIDEN e JONES, 2008). Mesmo com benefícios relatados do uso dessas técnicas de criatividade (SHNERDERMAN, 2007), a comunidade de engenheiros de software ainda pouco utiliza as técnicas de criatividade (SHNEIRDERMAN, 2007; COOK, 2010).

Este artigo apresenta o Guia de Padrões de Criatividade, um catálogo para suportar a geração de alternativas durante as fases de desenvolvimento de software. O guia contém uma seleção de técnicas de criatividade, transcrita e contextualizada aos cenários da engenharia de software. Para facilitar a adoção pelos desenvolvedores de software, as informações do guia são apresentadas obedecendo aos conceitos de Padrões de Projetos (ALEXANDER et al, 1977). Este trabalho apresenta o processo de desenvolvimento do Guia, seguindo as seguintes seções: $\mathrm{Na}$ seção 2 serão apresentados o referencial teórico que permeou todo o estudo, assim como a metodologia utilizada para a construção do Guia. Na seção de Conclusões será demonstrado o Guia e seu modo de utilização.

\section{DESENVOLVIMENTO}

\subsection{Referencial Teórico}

Ainda não há consenso na definição do que é "criatividade", mas há alguns pesquisadores que concordam que o termo é identificado como "resolução de problemas" (MAIDEN e JONES, 2008). Também é cientificamente aceito que a 
criatividade não é um dom, mas uma habilidade que pode ser aprendida, treinada e encorajada (NGUYEN e SHANKS, 2008). As técnicas de criatividade são ferramentas que estimulam a geração de alternativas (SÓLIS, 2010), através de uma grande variedade de técnicas e atividades (FOSTER e BROCCO, 2008; MUNARI, 1998; NORMAN, 2004; GRUBE e SCHMID, 2008).

Kirton (1989) identifica dois principais tipos de técnicas de criatividade: adaptação ou convergente e inovação ou divergente. O primeiro resolve problemas obedecendo a restrições do projeto, o segundo tende a redefinir o problema. As técnicas ainda podem ser praticadas individualmente ou em grupo (GRUBE e SCHMID, 2008a), utilizando atividades de combinação de elementos, livre associação, estímulos relacionados ou não ao problema, analogias e geração livre de alternativas (GRUBE e SCHMID, 2008b). Entender essas características ajuda na escolha da técnica mais apropriada para as pessoas e os problemas que temos em mão.

$\mathrm{Na}$ indústria de software, devido à sua necessidade de constante busca pela inovação, intensificaram as discussões sobre criatividade (ALVES, RAMALHO e DAMASCENO, 2007). Técnicas de criatividade podem auxiliar o desenvolvimento de software estimulando a geração de soluções criativas e inovadoras para os problemas enfrentados na área. Ainda que reconhecidamente importante no desenvolvimento de software, pouco tem sido estudado nessa área (GLASS, 2004). A exceção dessa regra é a fase de engenharia de requisitos, aonde poucos grupos vêm estudando notáveis aplicações dos conceitos durante as últimas décadas (LEMOS et al, 2012). Contudo, considerando o potencial e exponencial benefício da utilização de técnicas de criatividade no desenvolvimento de software, a comunidade de software ainda presta pouca atenção ao tema. Com isso em mente, construímos o Guia de Padrões de Criatividades, utilizando a estrutura dos Padrões de Projetos.

Padrões de Projetos é um tema proveniente da engenharia e adotado pela engenharia de software (GAMMA et al, 1995). Descrevem problemas comuns do desenvolvimento de software, soluções genéricas para tais problemas e consequência do uso das mesmas. Apresentam estrutura de informação semelhantes, fácil de entender e aplicar (GAMMA et al, 1995). Não são mutuamente excludentes, e alcançam melhores resultados se combinados (BRAZ, 2009). Tais características podem ser encontradas também nas técnicas de criatividade. Por esse motivo a utilização dos conceitos de Padrões de Projeto para o desenvolvimento do Guia de Padrões de Criatividade, um catálogo onde as técnicas de criatividade são explicadas, categorizadas e exemplificadas para o contexto de desenvolvimento de software.

\subsection{Metodologia}

Um dos maiores desafios de utilizar as técnicas de criatividade é identificar que técnica utilizar em determinado contexto (GRUBE e SCHMID, 2008). Cada fase de desenvolvimento de software e cada técnica de criatividade possuem características e objetivos distintos. O Guia de Padrões de Criatividade categoriza as técnicas de criatividade, de acordo com as necessidades específicas de cada fase de desenvolvimento de software, facilitando a escolha do projetista.

É necessário entender que a Engenharia de Software, geralmente, é dividida em fases e atividades bem peculiares. Vários são os modelos de engenharia de software, Modelo Cascata, Incremental, SCRUM, XP, RUP são as mais conhecidas (PRESSMAN, 2006). De todos esses modelos, o RUP foi utilizado como base para esse 
trabalho por possuir suas fases bem estruturadas e claramente descritivas às atividades que ocorrem em cada uma delas. As fases são: a) Análise e Projeto: Planejamento de toda a demanda que cerca a construção do sistema. Várias equipes de software trabalham em paralelo em diferentes funções do sistema; b) Definição de Requisitos: Entender os problemas do negócio e as características de informação, além do levantamento de requisitos; c) Modelagem: abrange três das principais fases modelagem do negócio, modelagem dos dados e modelagem dos processos - e estabelece o desenho do projeto para servir como base para as atividades de construção do software; d) Implementação: Durante esse estágio, o projeto de software é compreendido e como um conjunto ou unidades de programas. O uso de componente de software preexistente e a aplicação de geração automática de código; e) Testes e Implantação: Teste de unidades envolve verificar que cada unidade atende à sua especificação. Depois dos testes, o sistema de software é entregue ao cliente.

Cada fase possui um grupo de atividades específicas, essas atividades são realizadas para resolver um problema do projeto. As atividades podem ser apoiadas por diversas técnicas de criatividade. Compreender essas atividades foi o primeiro passo para o mapeamento de técnicas de criatividade ao longo das fases de ES.

\subsubsection{Seleção das técnicas de criatividade}

A fim de construir o Guia de Padrões de Criatividade, foi necessário selecionar quais técnicas de criatividade presentes na literatura melhor se encaixa em cada fase. O processo de seleção consiste nas seguintes fases:

Fase 1 envolve pesquisa ad-hoc em bibliotecas digitais e livros que discutem o sobre "criatividade" (BONFIM, NAGEL e ROSSI, 1977; MYCOTED, 2014; MIND TOOLS, 2014; GRUBE e SCHMID, 2008; MICHALKO, 2006). As técnicas encontradas na busca podiam ser encontradas em dois ambientes virtuais (mycoted.com, mindtools.org) e em um livro ("Thinkertoys", MICHALKO, 2006). Nessa fase foram identificados 254 técnicas de criatividade; A Fase 2 começa a análise dos objetivos e operações de cada técnica, assim como a eliminação daqueles que se apresentavam redundantes ou muito semelhantes a outros. Das técnicas muito semelhantes, eram escolhidas aquelas que possuíam as descrições mais completas de suas atividades. No fim, o número de técnicas foi reduzida para 98 . Na fase 3, foi avaliado quais técnicas de criatividade eram compatíveis com as fases de desenvolvimento de software. Primeiramente, foi escolhidas as técnicas que poderiam ser adaptadas para o contexto de engenharia de software, isto é, aquelas que eram voltadas para áreas específicas, como artes plásticas, moda e marketing foram removidas. No fim, o Guia de Padrões de Criatividade ficou com 60 técnicas de criatividade, listados na tabela 1.

\subsubsection{Critérios de Classificação}

As técnicas de criatividade foram classificados em 4 critérios: 1) Critérios de Controle, definido pelos estudos de Grube e Schmid (2008b), descreve se as técnicas necessitam o uso de algum artefato ou se só podem ser aplicados individualmente ou em grupo; 2) Critérios de Operação, classifica as técnicas de acordo com quatro tipos definidos por Boden (2009): exploração, combinação, transformação e validação; 3) Critérios de Abordagem, definido por Schmid e Grube (2008a), agrupam as técnicas de acordo com as seguintes atividades: livre associação, diferentes pontos de vista, lista de questões, estruturação, utilização de experts na área e entrada aleatória; 4) 
Critérios de Direcionamento, analisa o estilo das técnicas, ou seja, se possuem geração de ideias divergentes ou convergentes (BODEN, 2009; BROWN, 2010).

Tabela 1 - Lista final das técnicas de criatividade utilizadas na construção do Guia de Padrões de Criatividade

\begin{tabular}{llllll}
\hline 1 & 5 Why & 23 & Focus Group & 43 & Reciprocal Mode \\
2 & 635 Method & 24 & Free Association & 44 & Reverse \\
3 & 7x7 Technique & 25 & Future Fruits & & Brainstorming \\
4 & Anonymous Voting & 26 & Gap Analysis & 45 & Rolestorming \\
5 & Appreciation & 27 & Greeting Cards & 46 & S.O.D.A. \\
6 & Attribute Listing & 28 & Hall of Fame & 47 & SCAMPER \\
7 & Bionic & 29 & Heuristic Ideation & 48 & Sculptures \\
8 & Boundary Examination & & Technique & 49 & Search Conference \\
9 & Brainwritng & 30 & Ideabox & 50 & Similarities and \\
10 & Bullet Proof & 31 & Ideas Grid & & Differences \\
11 & Cartoon Story Board & 32 & Ideatoons & 51 & Simplex \\
12 & Cherry Split & 33 & Ishikawa Diagram & 52 & Six Thinking Hats \\
13 & Circle of Opportunity & 34 & Kinetics & 53 & Starbusting \\
14 & Clarification & 35 & Kneper and Tragoe & 54 & Stepladder Technique \\
15 & Clever Trevor & 36 & Matrix Reformulation & & Synectics \\
16 & Concept Fan & 37 & Mind Mapping & 55 & Storm Rice \\
17 & Dali Technique & 38 & Murder Board & 56 & Story Writing \\
18 & Dialectical Approach & 39 & NAF Technique & 57 & Super-hero \\
19 & Discussion 66 & 40 & Progressive Revelation & 58 & Talking Pictures \\
20 & Exaggeration & 41 & Provocation & 59 & Think Bubble \\
21 & Excursion & 42 & Rational Words & 60 & TRIZ \\
22 & False Faces & & & & \\
& & & & & \\
\hline & Fonte: Elaborado & & aut & & \\
\hline
\end{tabular}

Fonte: Elaborado pelo autor, com base na pesquisa realizada.

Por exemplo, a fase de levantamento de requisitos compreendem as seguintes atividades: identificação de stakeholders, reconhecimento de diferentes pontos de vista, identificação do problema, definição do escopo do projeto, e elaboração, negociação, documentação e validação dos requisitos (PRESSMAN, 2006). Cada uma dessas atividades foi mapeada sobre os critérios discutidos anteriormente. Este mapeamento foi realizado a partir das análises de literatura sobre Criatividade e Ciências da Computação, procurando observações e estudos que auxiliassem no mapeamento. 

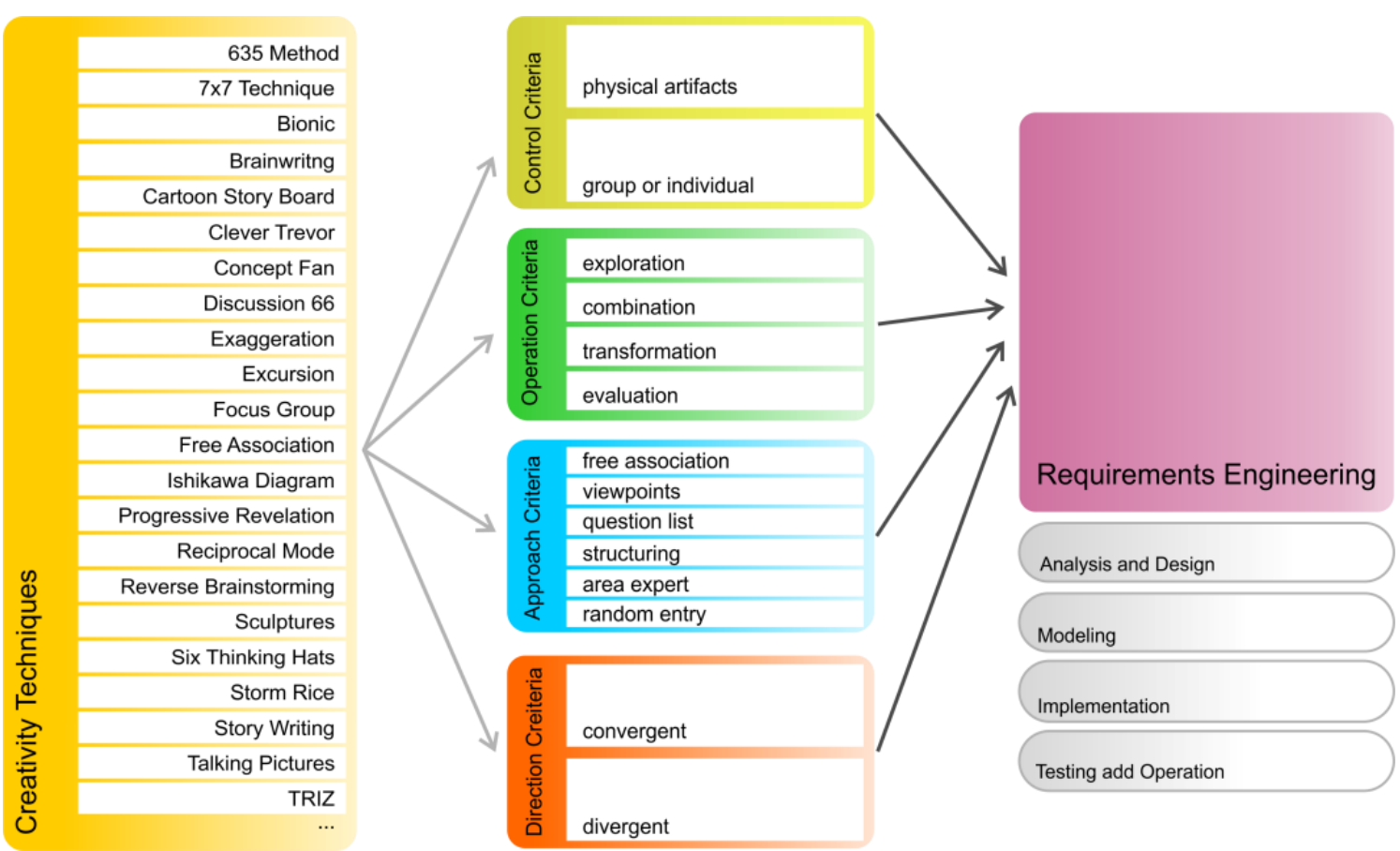

Figura 1 - Exemplo do processo percorrido durante o mapeamento das técnicas de criatividade às fases de desenvolvimento de software.

Esse estudo pode ser exemplificado quando Pressman (2006) observa que os requisitos podem ser levantados através de reuniões ou entrevistas individuais (MAIDEN et al, 2010), o que sugere que as técnicas classificadas como "Grupos" e "Individuais" podem ser utilizadas nessa fase. Pressman também afirma que combinação e transformação de ideias através de múltiplos pontos de vista são importantes para a elaboração dos requisitos (PRESSMAN, 2006). Baseado nessa informação, inferimos que as técnicas classificadas como "transformação" e "Combinação" se encaixam nesta fase.

Esta análise da fase de levantamento de requisitos continua através das pesquisas Maiden, Robertson e Robertson (2006), Kirton (1989) e Pressman (2006). Podendo se estender, utilizando a mesma acuidade, para as outras fases de desenvolvimento de software. Um exemplo do caminho percorrido pelo mapeamento das técnicas de criatividade pode ser observado na figura 1 . É um fato que o mapeamento realizado é baseado na interpretação, observação e experiências dos pesquisadores em engenharia de software e criatividade. Reconhecendo que o mapeamento é passível de outros resultados, se elaborados por uma equipe multidisciplinar. A validação empírica e teórica do mapeamento foi realizada e os resultados podem ser encontrados em Vieira (2012) e Vieira, Alves e Duboc (2012). Porém, este artigo possui o recorte descritivo apenas a descrição dos processos de desenvolvimento do Guia de Padrões de Criatividade.

\section{CONCLUSÃO}

O Guia de Padrões de Criatividade foi desenvolvido para encorajar e dar suporte a desenvolvedores de software que queiram utilizar técnicas de criatividade em seus projetos. Para esse fim, foi necessário trazer as técnicas de criatividade para 
uma linguagem comum aos desenvolvedores de software. Assim, traçamos as seguintes estratégias: 1) adotar uma estrutura familiar aos desenvolvedores de software na descrição das técnicas, no caso, a estrutura de Padrões de Projeto; 2) exemplificar a utilização das técnicas em problemas próprios da indústria de software; 3) aproximar a linguagem e os termos utilizados nas técnicas para o contexto de engenharia de software e; 4) utilizar uma interface atrativa e dinâmica, que facilitasse a utilização das técnicas.

O Guia seguiu a mesma estrutura e princípios utilizados pelos Padrões de Projeto. Onde é claramente descrito um problema de desenvolvimento de software e apresentado todas as informações necessárias para a resolução da mesma através daquela técnica de criatividade. É importante lembrar que, assim como os Padrões de Projetos, as técnicas de criatividades alcançam melhores resultados se utilizados combinadas. A seguir está descrita as seções presentes no Guia de Padrões de Criatividade:

1) Nome: descreve a essência da técnica com um curto e expressivo nome. Os nomes das técnicas não foram modificados de acordo com a literatura geral sobre criatividade;

2) Objetivo: Descreve de modo conciso o objetivo principal da técnica, auxiliando o usuário na seleção das técnicas;

3) Exemplo: Apresenta um exemplo de problemas que podem acontecer em um ambiente de desenvolvimento de software, descrevendo como a técnica pode resolver aquele problema;

4) Direcionamento das Ideias: está seção não tem paralelo nos Padrões de Projeto. Descreve se técnica de criatividade possui atividades que suportem o pensamento divergente ou convergente;

5) Aplicabilidade: Apresenta as situações onde a técnica é aplicada. Identificam quais fases de ES a técnica é direcionada e lista cenários que a técnica pode ser aplicada. Considerando que cada fase possui várias técnicas recomendadas, essa seção apresenta-se como filtro para a seleção das técnicas de criatividade;

6) Participantes: Nesta seção são descritos os critérios de controle das técnicas de criatividade, ou seja, a quantidade de pessoas que participam da técnica e os apetrechos físicos que deverão ser utilizadas. Técnicas de criatividade podem ser utilizadas em grupos ou de forma individual. Nesta sessão o usuário identificará a quantidade de pessoas recomendada para a execução da técnica;

7) Mais Informações: dados bibliográficos e informações interessantes sobre a técnica de criatividade;

8) Símbolos: Os símbolos fazem referencia às fases de desenvolvimento de software a qual aquela técnica é recomendada; 
9) Tempo: aqui é descrito a quantidade de tempo necessário para aplicar a técnica. É dividida em "Curto" (não mais que 40 minutos), "Médio" (de uma a três horas) e "Longo" (mais de três horas).

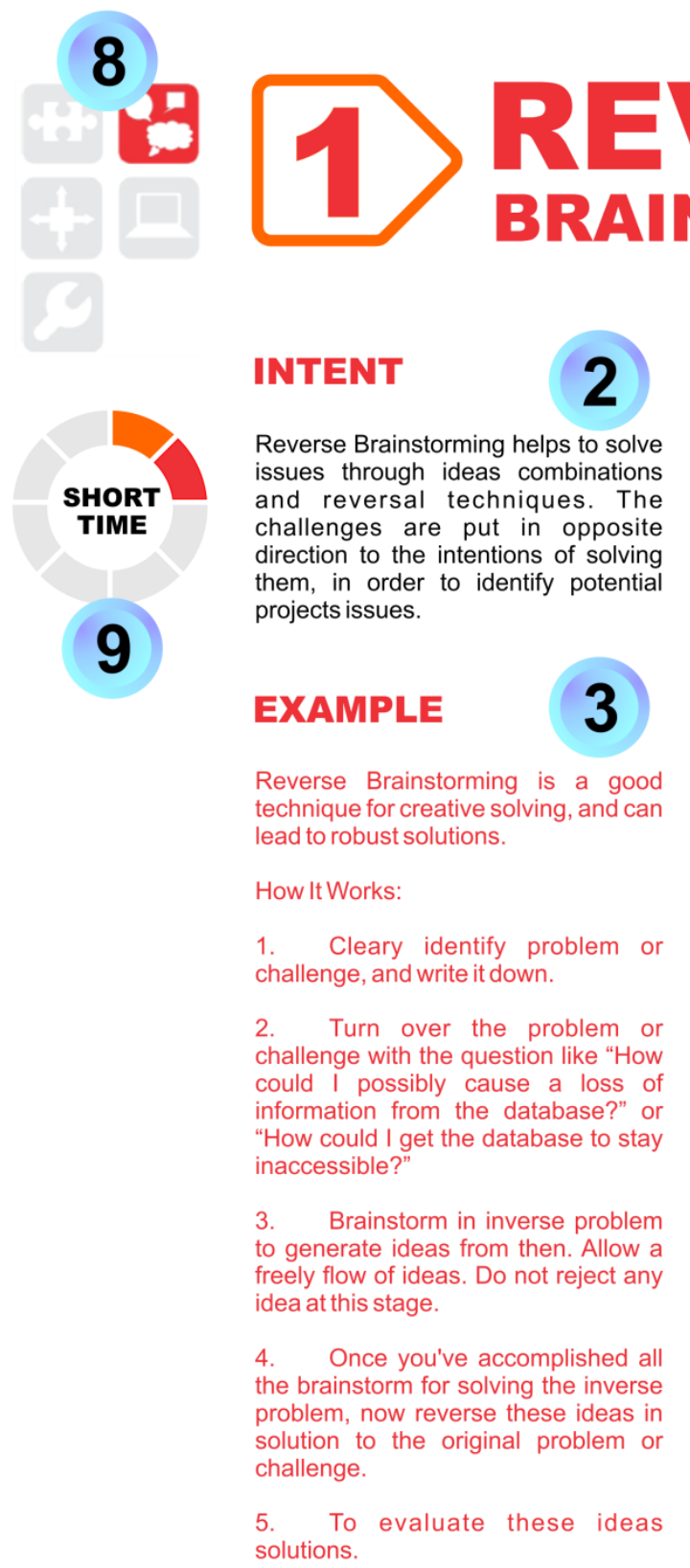

\section{IDEA DIRECTION 4 \\ Convergent and/or Divergent \\ APPLICABILITY

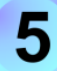 \\ Requirements Definition Phase \\ Requirements Definition: Putting the challenge in opposite direction, force the techniques users to identify recurring problems and find creative answer. At stage of identifying requirements, the technique can help participants to see problems, solutions and functions outside the scope of their previous knowledge, however, relevant to the project. \\ PARTICIPANTS 6}

Can be used in groups or alone, do not need physical tools.

\section{MORE INFORMATION}

Operation: exploration, combination.

Approach: question list.

Figura 2 - Exemplo de uma página do Guia de Padrões de Criatividade, enumerados os elementos e seções presentes na página.

O Guia de Padrões de Criatividade também está representado em um ambiente virtual (https://sites.google.com/site/guiadecriatividade/home), onde é possível 
encontrar todas as 60 técnicas de criatividade obedecendo aos mesmos princípios de interface e estrutura.

\subsection{Considerações Finais}

Este artigo apresenta o Guia de Padrões de Criatividade, um artefato desenvolvido para auxiliar os desenvolvedores de software a adotar o uso de técnicas de criatividade em seus projetos. O Guia possui estruturas e princípios semelhantes aos Padrões de Projeto (GAMMA et al, 1995), para exemplificar e explicar as técnicas no contexto de desenvolvimento de software. De fato, a contribuição chave desse projeto é o encapsulamento de um montante de conhecimento sobre criatividade e técnicas de criatividade, utilizando uma estrutura padronizada e conhecida por desenvolvedores de software, vocabulário adequado ao contexto e construção de exemplos comuns aos cenários de desenvolvimento de software.

O Guia de Padrões de Criatividade se apresenta como uma importante ferramenta para o estímulo à criatividade em um mercado onde a inovação é princípio vital. Desenvolvida para que as equipes de desenvolvimento de software não dependam de "especialistas em criatividade" para executar e exercitar técnicas de criatividade. Reduzir as amarras para a criação é ponto fundamental para a inovação e objetivo maior do Guia.

Estudos empíricos sobre as vantagens de utilização de Guia de Padrões de Criatividade foram realizados (VIEIRA, 2012; VIEIRA, ALVES e DUBOC, 2012), obtendo respostas muito positivas ao uso do artefato. Aceitação da interface, estrutura e princípios do Guia foi constante durante os estudos aplicados em projetos reais. Contudo, mais pesquisas necessitam ser realizadas para que seja possível verificar a eficácia do Guia em todo o ciclo de vida do desenvolvimento de software, em um número grande de projetos, de variados tamanhos e objetivos.

\section{REFERÊNCIAS}

ALVES, C.; RAMALHO, G.; DAMASCENO, A. Challenges in Requirements Engineering for Mobile Games Development: The Meantime Case Study. Requirements Engineering Conference, 2007.

BODEN, M. A. Computer models of creativity. Al Magazine 30(3): 23-34. AAAI Press. 2009.

BOMFIM, G. A.; NAGEL, K.D.; ROSSI, L.M. Fundamentos de uma metodologia para desenvolvimento de produtos. COPPE/UFRJ, 1977.

BRAZ, S. A Qualitative Assessment of Modularity in CaesarJ components based on Implementations of Design Patterns. 2009. M.Sc. thesis, Universidade Nova de Lisboa.

BROWN, Tim. Design Thinking. Rio de Janeiro: Elsevier, 2010.

FOSTER, F.; BROCCO, M. Understanding Creativity-Technique Based Problem Solving Processes. In: I. Lovrek, R.J. Howlett, and L.C. Jain (Eds.): KES 2008, Part II, LNAI 5178, Berlin Heidelberg, 2008. 
GAMMA, et al. Design Patterns: Elements of ReusableObject-Oriented Software. Addison-Wesley, Reading, MA. 1995.

GLASS, R. L. Software Creativity 2.0. Developer *Books. 2006.

GRUBE, P.; SCHMID, K. Selecting Creativity Techniques for Innovative Requirements Engineering. Third International Workshop on Multimedia and Enjoyable Requirements Engineering. 2008a.

GRUBE, P; SCHMID, K. State of Art in Tools for Creativity. idSpace Project. University of the Netherlands. 2008b.

KIRTON, M. J. Adaptors and innovators: Sfyfes of creativity and problem solving. London: Routledge. (ed.) 1989.

LEMOS, J. et al. A Systematic Mapping Study on Creativity in Requirements Engineering. In: ACM Symposium of Applied Computing, 2012.

MAIDEN, N.; JONES, S. Provoking Creative Design: Making it Scale. British Computer Society, 2008.

MAIDEN, N.; ROBERTSON, S. E ROBERTSON, J. Creative Requirements: Invention and Its Role. In Requirements Engineering, ICSE'06, (2006).

MAIDEN, Niel, et al. Requirements Engineering as Creative Problem Solving: A Research Agenda for Idea Finding. 18th IEEE International Requirements Engineering Conference (RE), Sydney, 2010.

MIND TOOLS. Disponível em: <http://www.mindtools.com>. Acessado em: 12 mar. 2014.

MUNARI, B. Das coisas nascem coisas. Martins Fontes, 1998.

MYCOTED. Disponível em: <http://www.mycoted.com>. Acessado em: 12 mar. 2014.

NGUYEN, S.; SHANKS, G. A framework for understanding creativity in requirements engineering. Information and Software Technology, 51. Elsevier. 2008.

NORMAN, D. A. Emotional Design: Why do We Love (Or Hate) Everyday Things. Basic Books. 2004.

POTTS, C. Invented Requirements and Imagined Customers: Requirements engineering for Off-the-Shelf software., Second International Symposium on Requirements Engineering, 1995.

PRESSMAN, R. Software Engineering. 6th Edition: Mcgraw Hill, 2006.

SHNEIDERMAN, B. et al. Creativity Support Tools: Accelerating Discovery and Innovation. Communications of the ACM, Vol. 50, № 12, 2007.

SOLÍS, C.; ALI, N. Distributed Requirements Elicitation Using a Spatial Hypertext Wiki. IEEE International Conference on Global Software Engineering. 2010.

VIEIRA, Elton. Guia de Padrões de Criatividade: Um apoio à aplicação de técnicas de criatividade em projetos de desenvolvimento de software. 2012. Dissertação (Mestrado), Universidade Federal de Pernambuco, Pós-graduação em Ciências de Computação. 
VIERA, Elton; ALVES, Carina; DUBOC, Letícia. Creativity Patterns Guide: Support for the application of creativity techniques in Requirements Engineering. In IFIP, HumanCentred Software Engineering Conference. HCSE'2012. Springer Science \& Business Media, 2012. 